\title{
Applications of Extended Reality (XR) for Creation of Real-and-Virtual Combined Environments and Human
}

\author{
Arihant Chhajer ${ }^{1}$ \\ ${ }^{1}$ B.Tech, Biotechnology, Jaipur National University, Jaipur, Rajasthan \\ 1 arihantmparcs@gmail.com
}

\begin{abstract}
The Technological pace is increasing day by day where artificial intelligence along with the extended reality and other technologies has emerged hugely. The new industrial world, especially the entertainment and gaming industry, has adopted these technologies on a high scale. Here, in this article the key concept of extended reality has been described. This paper has also mentioned their unique characteristics and how it works in describing manner. The major purpose of this study is to make the readers understand about the immerse feature of XR. Significant discussion in this study has focused on the creation of a unique blend of real and virtual world addressing the role of humans in this technology.
\end{abstract}

Keywords - Artificial Intelligence, Virtual Reality, Augmented Reality, Mixed Reality

\section{Introduction}

Extended reality (XR) is the significant term which incorporates real and virtual environments that are generated through wearable devices or by the computer technology in order to offer immersive experiences. The term is also described as the set of all the immersive technologies that merge the real and the virtual world at one place. Here, in this study the concept of extended reality is explained. This article also encompasses the application of extended reality (XR) to create the proper combination of real and virtual world correlating humans. Major aim of this study focuses on the discussion of key features of XR. Defining all those characteristics the articles set its purpose to give better understanding about the topic.

\section{Literature review}

\section{Concept of XR}

Both the number of advancement and XR applications with these applications is growing day-by-day. The growth of this technology is ongoing as it is still evolving to develop machine-human interaction. XR encompasses three major pillars of unique and immersive technology VR (Virtual Reality), AR (Augmented Reality), and MR (Mixed Reality). This unique and immersive technology includes HMD (Head Mounted Display) to offer immersive expression by simulated environment (Kerdvibulvech and Guan, 2019). The "simulated digital environment" makes the user assume if they are present in that exact place. An example can clear the aspect- VR developers can develop an artificial world which makes those users feel like they are standing around the Jupiter. The entertainment and gaming industries have adopted this unique technology at high scale. In the WR component, various smart devices are used to explore the extraordinary sound system and digital graphics in this real world. Some of such kind of digital details combined or overlaid into this real world surroundings such as animation, images and texts (Gironacci et al. 2020). These enhanced features can be achieved using multiple electronic devices like smart phones, tables, screens, AR screens or the normal screen. Today the entertainment industries are using these features hugely to provide some extraordinary features in their production. The popularity of such immerse technology has become like a revolutionary manner which creates an extraordinary combination of artificial world and real environment regarding the human experiences. 
Integrating Researchers to Incubate Innovation

\section{Working Procedures of extended Reality}

From the perspective of the developers, extended reality is acquired by organizing immersive algorithms. Data scientists and software developers should design useful and efficient algorithms to create interesting and extraordinary XR features which offer such a unique experience to the users. Another important concept regarding this XR requires it to be incorporated by those immersive technology developers including advanced computerized vision and 3D modeling. Artificially featured programs are produced using unique biomechanical technologies, motion learning, and machine learning (Fast-Berglund et al. 2020). Some of these programming languages which are included in the design of XR are C, JavaScript, Java, Swift, and Python. Full description of such languages and important AR application can be observed here (Doolani et al. 2020). Developers who are interested to incorporate such applications in programming in VR development can gain more information in this place.

From the user's perspective three major XR components perform an implicative role to offer immersive experiences. With the solution of AR, Smartphone cameras usually capture these environments in recognizing specific points where more information will be included. Digital information of computer-generated information is then overspread onto those environments that are captured using markers. Such trackers- GPS, laser, and other sensors can also be incorporated to overlay the information over the aforementioned environment. MR functions just like the AR, although MR offers more immersive and exemplified experiences than XR allowing mixed interaction of real worlds and virtual worlds.

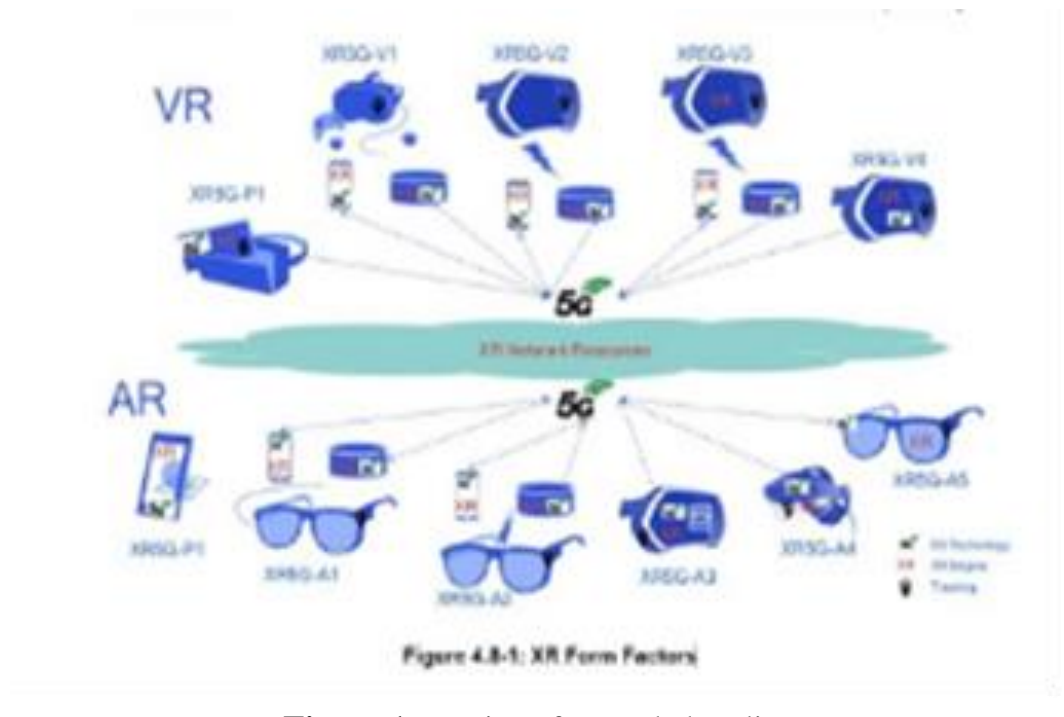

Figure 1: Device of extended reality

(Source: Inspired by Kerdviebulvech and Guan, 2019)

Virtual reality actually relies on the human senses offering unique experiences that are different from the real world (Fadzli et al. 2019). The major components used are sight, touching the hearing features, and also the power of imagination and mind. Multiple VR features offer the users the measures for reconfiguration. In the midst of the cases forgetting this kind of experience the use of a headset is necessary to ignore outer influences because the human sense organs are also important as well as the unique features of the technologies.

\section{Use of XR for creation of real-and virtual combined environment and human 200}

The Extended reality (XR) defers the entire real and virtual environment generated by technological innovation and wearables. According to Ziker et al. (2021), Augmented reality (AR) is essential to make an attachment between the real world and the virtual world. The XR is needed to make a combination between the virtual world and environment, it is significantly important to make an interaction between the human and computer technologies. The author has systematically described the relation between AR, Virtual reality (VR) and Augmented Virtuality (AV) for focusing on Mixed Reality (MR). This technology is essential to enhance the productivity of manufacturing technologies. Moreover, it is significantly important to make an employee's engagement, wellness and performance. Moreover, XR innovation is significantly important to implement the Internet of things (IoT), smart manufacturing and cloud manufacturing for the enhancement of productivity.

The use of XR is significantly important to increase cognitive skills among humans. The development of close integrated technologies, automated designs and robotic structure is beneficial for the maintenance of the working efficiency to enhance productivity. Proper assessment, frequent monitoring and cognitive performances are important for the reduction of the labour cost in the manufacturing industry. 


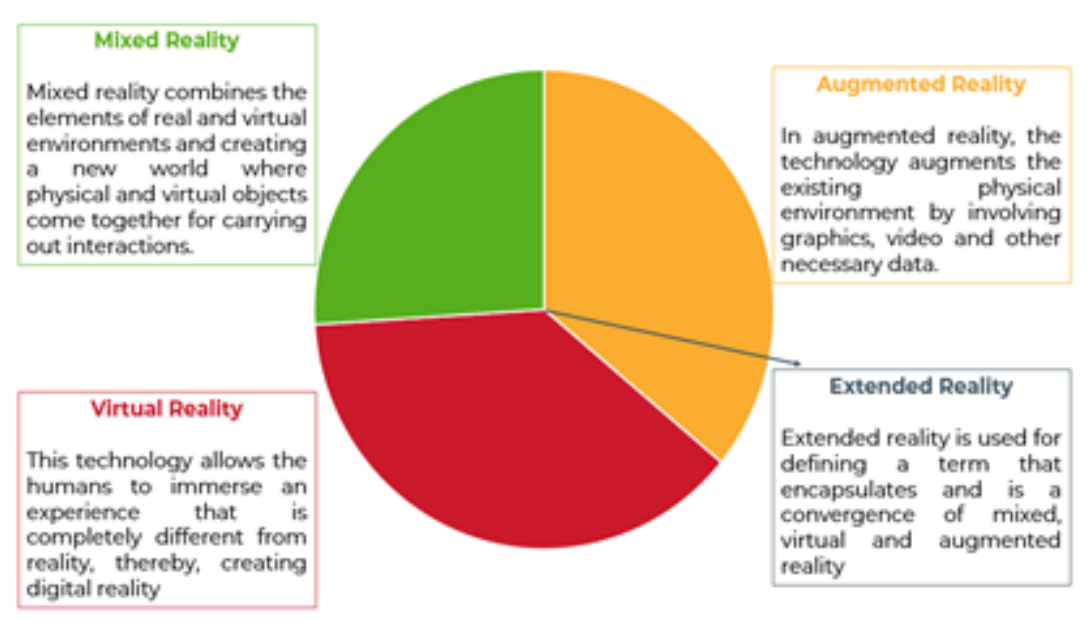

Figure 2: Uses of XR to enhance productivity

\section{Method and materials}

(Source: Doolani et al. 2020)

The research article has been conducted on the XR technologies for making the interaction between machines and humans. Some effective peer-reviewed journals have been selected to enhance knowledge on this topic. According to Doolani et al. (2020), identification of the inclusion and exclusion factors helped to focus on the key factors of XR. Focusing on the keywords helped to find out the appropriate journals on the XR. Nearly 12 journals have been selected to acquire information. Five years of journals have been selected to collect appropriate and correct information on the researched topic. The secondary data collection process has been used to acquire information on the research topic. All qualitative and qualitative data has been collected on the XR to focus on real and virtual world interaction for making human-machine interaction. The selection of appropriate journals helped to focus on the importance of XR. Journals with insufficient data have been rejected for constructing this research article. All selected journals have been collected from google scholar. A systematic method has been used to complete this research article with authenticity.

\begin{tabular}{|c|c|}
\hline Inclusion criteria & $\begin{array}{c}\text { Related publications, simple English languages, peer-reviewed journals and } \\
\text { authentic information on the XR }\end{array}$ \\
\hline Exclusion criteria & $\begin{array}{c}\text { Spelling errors, other the English languages, political interferences, sexual abuses, } \\
\text { sensitivity, other than English languages. }\end{array}$ \\
\hline
\end{tabular}

\section{Discussion}

Table 1: Inclusion and exclusion criteria

As mentioned, in the literature review portion, the $\mathrm{X}$ is denoted as the $\mathrm{X}$ reality for representing the future spatial computing technologies, such as MR, AR and VR. According to Stoynov et al. (2021), it is significantly important for offering innovative and effective safety training to enhance proactivity in the workplace. Moreover, it provides the opportunity to develop professional practices for making significant changes in infrastructural development. It has numerous advantages to enhance the organisation's economic development. In the opinion of Georgiev. and Casakin (2020), reproducing the working environment is significantly important to maintain interactivity among the workers. In case of emergency jobs, such as pilot, airline and surgeon, XR helps to reinvent innovative techniques for resolving any kinds of critical situations in the workplace. 
Integrating Researchers to Incubate Innovation

Moreover, the XR is significantly important to motivate the learners by providing graph charts. It is significantly important to determine the growth rate of the employee's and student's performances.

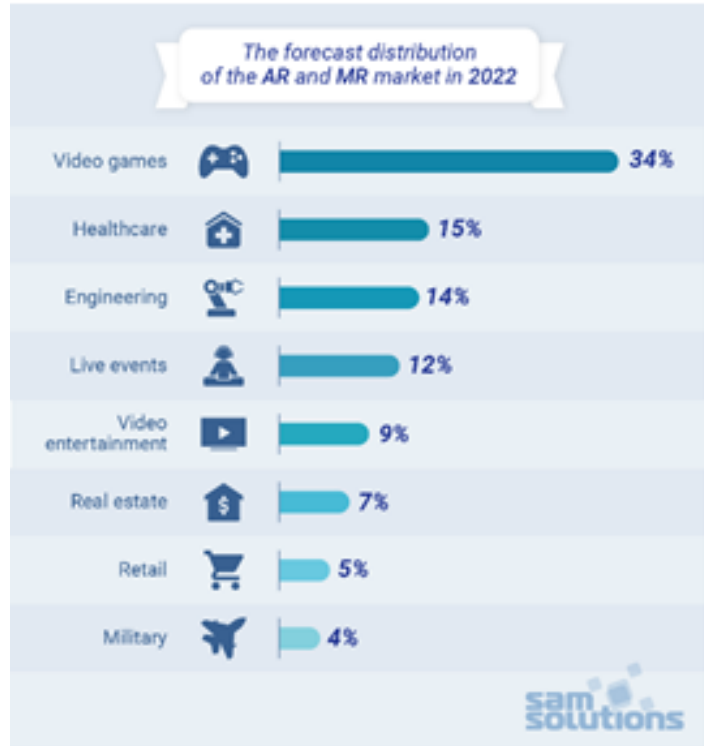

Figure 3: distribution of the AR and MR

(Soyrce: to Fadzli et al. 2020)

Apart from these, XR innovation is essential to involve innovative stages to adopt appropriate designs with real objects. Implementation of the Computer-aided design software was important to optimise the appropriate design and make frequent changes in the manufacturing process. According to Fadzli et al. (2020), it is significantly important for the development of the design replica in the technical system, such as assembly line, industrial process and machines. Apart from these, making virtual prototypes is significantly important for the reduction of design cost and labour costs. Moreover, it is essential to make fluency in the entire manufacturing process for reducing time consumption.

According to the periodic research, many estate agents and property developers have the tendency to use this strategy to interact with the people. According to Raybourn et al. (2019), the use of the AI-powered XR app is beneficial to determine different types of interfaces to enhance personal experiences. Moreover, it provides the opportunity to identify the potential employees for enhancement of the sales rate (Stubbemann et al. 2021). The XR has the potential to maintain transparency by identification of any kinds of conflicts or barriers in the manufacturing process. According to the selected researched articles, the XR is enabled to provide 360-degree pictures, for a faithful representation to the consumers. Moreover, this feature is significantly important for providing an interior design of the products. In addition to this, AR is significantly important for representing the 2D and 3D elements from virtual reality and the real environment. The use of haptic devices, monitor sensors and location sensors is significantly important to communicate or interact with the technical devices.

Apart from these, the Human-machine interface (HMI) and 3D audio are useful to communicate and control the entire system to enhance the interaction capabilities. According to Margetis et al. (2021), the location sensor is useful to make crucial decisions for the nation. In addition to this, the implementation of the MR is enabled to provide better experiences to the consumers. According to the research paper, it is significantly important for developing secure programmes to enhance productivity.

\section{Recommendation and conclusion}

\section{Recommendation}

The recommended section will provide some innovative strategies for better incorporation of the XR to organise all factors in a systematic way. Moreover, it is essential to drive productivity and efficiency in the workplace.

Firstly, the initial approach is required to get long term success in the IT sector. A clear and concise plan and proper experiment is required to get fruitful outcomes. Moreover, it could be effective to avoid failure throughout this process. 
In addition to this, it requires sufficient resources and enough preparation to start the XR for appropriate enterprise settings. It helps to enhance the value of XR for the enhancement of the activity.

Apart from these, data accumulation is needed to eradicate the errors. According to Margetis et al. (2021), implementation of the Key Performance Indicator (KPI) is required to focus on the growth rate. Implementation of different XR analytic tools helps to maintain punctuality, safety, First timFirst-time Quality (FTQ) and many others.

\section{Conclusion}

The research article focused on the XR to create real-and-virtual combined environments and human-machine interaction. The introduction has shades light on the concept of the XR. This literature review has provided detailed information from the existing literature. Different XR technologies, key features, concepts and uses of XR have been discussed. The methods and materials have discussed the inclusion and exclusion criteria to choose appropriate journals from the discussion. The recommendation has recommended effective strategies for the improvement of the XR to enhance productivity.

\section{References}

1. Doolani, S., Wessels, C., Kanal, V., Sevastopoulos, C., Jaiswal, A., Nambiappan, H. and Makedon, F., 2020. A review of extended reality (xr) technologies for manufacturing training. Technologies, 8(4), p.77.

2. Doolani, S., Wessels, C., Kanal, V., Sevastopoulos, C., Jaiswal, A., Nambiappan, H. and Makedon, F., 2020. A review of extended reality $(\mathrm{xr})$ technologies for manufacturing training. Technologies, 8(4), p.77.

3. Fadzli, F.E., Kamson, M.S., Ismail, A.W. and Aladin, M.Y.F., 2020, November. 3D telepresence for remote collaboration in extended reality (xR) application. In IOP Conference Series: Materials Science and Engineering (Vol. 979, No. 1, p. 012005). IOP Publishing.

4. Fadzli, F.E., Kamson, M.S., Ismail, A.W. and Aladin, M.Y.F., 2020, November. 3D telepresence for remote collaboration in extended reality (xR) application. In IOP Conference Series: Materials Science and Engineering (Vol. 979, No. 1, p. 012005). IOP Publishing.

5. Fast-Berglund, Å., Gong, L. and Li, D., 2018. Testing and validating Extended Reality (xR) technologies in manufacturing. Procedia Manufacturing, 25, pp.31-38.

6. Georgiev, G.V. and Casakin, H., 2020, May. Mitigating design fixation with evolving extended reality technology: An emerging opportunity. In Proceedings of the Design Society: DESIGN Conference (Vol. 1, pp. 1305-1314). Cambridge University Press.

7. Gironacci, I., Vincs, K. and McCormick, J., 2020, February. A Recommender System of Extended Reality Experiences. In Proceedings of the 2020 3rd International Conference on Image and Graphics Processing (pp. 96-100).

8. Kerdvibulvech, C. and Guan, S.U., 2019, July. Affective Computing for Enhancing Affective Touch-Based Communication Through Extended Reality. In International Conference on Computational Science and Its Applications (pp. 351-360). Springer, Cham.

9. Margetis, G., Apostolakis, K.C., Ntoa, S., Papagiannakis, G. and Stephanidis, C., 2021. X-Reality Museums: Unifying the Virtual and Real World Towards Realistic Virtual Museums. Applied Sciences, 11(1), p.338.

10. Raybourn, E.M., Stubblefield, W.A., Trumbo, M., Jones, A., Whetzel, J. and Fabian, N., 2019, July. Information design for xr immersive environments: Challenges and opportunities. In International Conference on Human-Computer Interaction (pp. 153-164). Springer, Cham.

11. Stoynov, V., Ivanov, A. and Mihaylova, D., 2021. Conceptual framework for quality assessment in human-centric 6G XR services. In IOP Conference Series: Materials Science and Engineering (Vol. 1032, No. 1, p. 012009). IOP Publishing.

12. Stubbemann, L., Dürrschnabel, D. and Refflinghaus, R., 2021, May. Neural Networks for Semantic Gaze Analysis in XR Settings. In ACM Symposium on Eye Tracking Research and Applications (pp. 1-11).

13. Ziker, C., Truman, B. and Dodds, H., 2021. Cross reality (XR): Challenges and opportunities across the spectrum. Innovative Learning Environments in STEM Higher Education, p.55. 